\title{
IACR: an interference-aware channel reservation for wireless sensor networks
}

\author{
Sangsoon Lim \\ Department of Computer Engineering, Sungkyul University, Anyang, South Korea
}

\begin{abstract}
Article Info
Article history:

Received May 5, 2018

Revised Sep 27, 2018

Accepted Oct 12, 2018

\section{Keywords:}

Channel reservation

Energy efficiency

Interference

Wireless sensor networks

ABSTRACT

In battery-based wireless sensor networks, energy-efficient operation is one of the most important factors. Especially, in order to improve energy efficiency in wireless sensor networks, various studies on low power operation have been actively conducted in the MAC layer. In recent years, mutual interference among various radio technologies using the same radio frequency band has become a serious problem. Wi-Fi, ZigBee, and Bluetooth use the same frequency band of $2.4 \mathrm{GHz}$ at the same time, which causes various signal interference problems. In this paper, we propose a novel channel reservation scheme, called IACR, to improve the energy efficiency of wireless sensor networks in an environment where interference occurs between various wireless technologies. The proposed scheme inserts a PN code into a long preamble for exchanging transmission status information between a transmitting node and a receiving node, thereby improving the transmission success probability while receiving less influence on transmission of other radio technologies. We performed an event-driven simulation and an experiment to measure the signal detection rate. As a result, it can be seen that the proposed technique reduces the packet drop rate by $15 \%$ and increases the discoverable distance of the control packet for channel reservation.
\end{abstract}

Copyright $@ 2019$ Institute of Advanced Engineering and Science. All rights reserved.

\section{Corresponding Author:}

Sangsoon Lim,

Department of Computer Engineering,

Sungkyul University,

Anyang, South Korea.

Email:1ssgood80@gmail.com

\section{INTRODUCTION}

Since Wireless Sensor Networks (WSNs) consist of battery-powered sensor nodes, energy efficiency is one of the most important performance criteria. Particularly, it is known that idle listening waiting for receiving a potential packet and overhearing for listening to a packet transmitted to another node are the main energy wasting factors [1]. Various Media Access Control (MAC) protocols have been proposed [2-5] to mitigate excessive energy consumption problems. These solutions utilize a duty-cycling technique that repeats the operation of periodically turning off and on the RF module of the sensor node. The duty-cycling technique is divided into two types of approaches: synchronous and asynchronous approach.

The synchronous approaches [2], [3] broadcast control packets periodically to all sensor nodes in the vicinity, and informs the duty-cycling period in advance, so that the cycles of all the nodes are equalized. Therefore, the idle listening time can be minimized by turning off the RF module in the absence of packet transmission. However, synchronizing all sensor nodes is not a trivial issue in a large scale WSNs. On the other hand, since the asynchronous approches [4], [5] do not require synchronization between the sensor nodes, the approach can be applied to a large scale network. For this reason, we mainly focus on the asynchronous approach in this paper. In the case of the asynchronous approach, a long preamble-based asynchronous rendezvous technique between transmitters and receivers effectively reduces idle listening [4]. 
However, these approaches cannot handle diverse interference scenarios in the wireless coexistence environment.

Due to the recent rapid increase in the use of small mobile devices, the ISM band (Industrial, Scientific and Medical Band) of the $2.4 \mathrm{GHz}$ band has been developed by a variety of wireless technologies such as Wi-Fi, Bluetooth and ZigBee. When different wireless technologies exist in the same frequency band according to respective standards, different wireless technologies are inevitably not recognized each others due to different PHY/MAC characteristics. At this time, serious interference may occur between the node attempting transmission and the node not acknowledging transmission, which may cause transmission failure. This problem is defined as Cross-Technology Interference (CTI) problem [6]. When the sensor nodes performing a low power operation exchange their information, they severely suffer from the interferences of Wi-Fi networks.

In this paper, we propose an intelligent channel reservation scheme, called IACR, to improve the overall performance of both low-power ZigBee networks and Wi-Fi networks. When the sensor node periodically wakes up, it can detect ZigBee transmissions with their exact wake-up schedules in the presence of other wireless interferences. Our proposed scheme provides robust channel reservation for a low-power ZigBee node. Although there exist different wireless technologies, a ZigBee node can identify the reservation period of the ZigBee transmission with an embedded PN code. Thus, the ZigBee nodes can avoid severe packet collisions and false-wake ups caused by Wi-Fi packet transmissions. We evaluate the performance of packet delivery ratio and energy consumption via an event-driven simulation.

The rest of this paper proceeds as follows. Section 2 introduces the related work. We then describe our channel reservation scheme in Section 3. Section 4 evaluates the proposed scheme. Finally, Section 5 concludes the paper.

\section{RELATED WORK}

\subsection{Low power operations of wireless sensor networks}

As shown in Figure 1 the transmitting node of the basic asynchronous method [4] transmits a packet to the neighboring sensor nodes within a single-hop period, called polling interval. The receiving node awakes the RF module once every channel polling period and keeps awake until receiving a packet if it recognizes through the long preamble that there is a packet being sent. However, this scheme causes an unnecessary reception interval due to excessive preamble. In addition, the energy consumption of neighbor nodes is also increased due to overhearing problem.

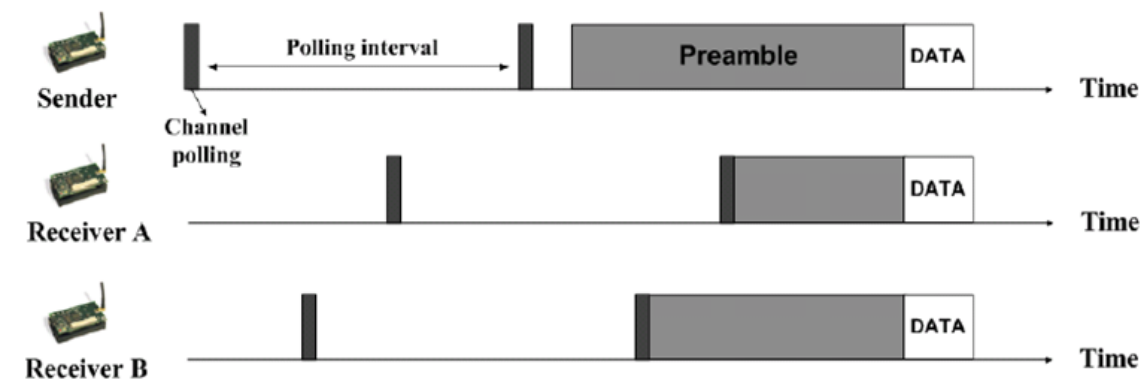

Figure 1. The basic operation of a general asynchronous approach

To solve overhearing problems due to excessive preambles, an ACK-based asynchronous method has been proposed [5]. As shown in Figure 2 the ACK-based asynchronous method uses a short preamble segment and an early ACK packet. Each short preamble has the address of the destination node. The receivers compare with the destination address of the short preamble when waking up after the channel polling time and confirming the packet to be transmitted to the receiver node. If it is not a packet to itself, it will immediately turn off the RF module and reduce its energy consumption to avoid meaningless overhearing. If it is a packet coming to itself, it sends an early ACK to the transmitter to stop sending the prepreamble and requests to send the actual packet. As a result, the overhead problem can be mitigated through the short preamble negotiation process, and the propagation delay in a single hop unit can be significantly reduced. However, if the nodes cannot correctly receive short preambles or an early ACK in the presence of $\mathrm{Wi}-\mathrm{Fi}$ interferences, they consume much energy. 


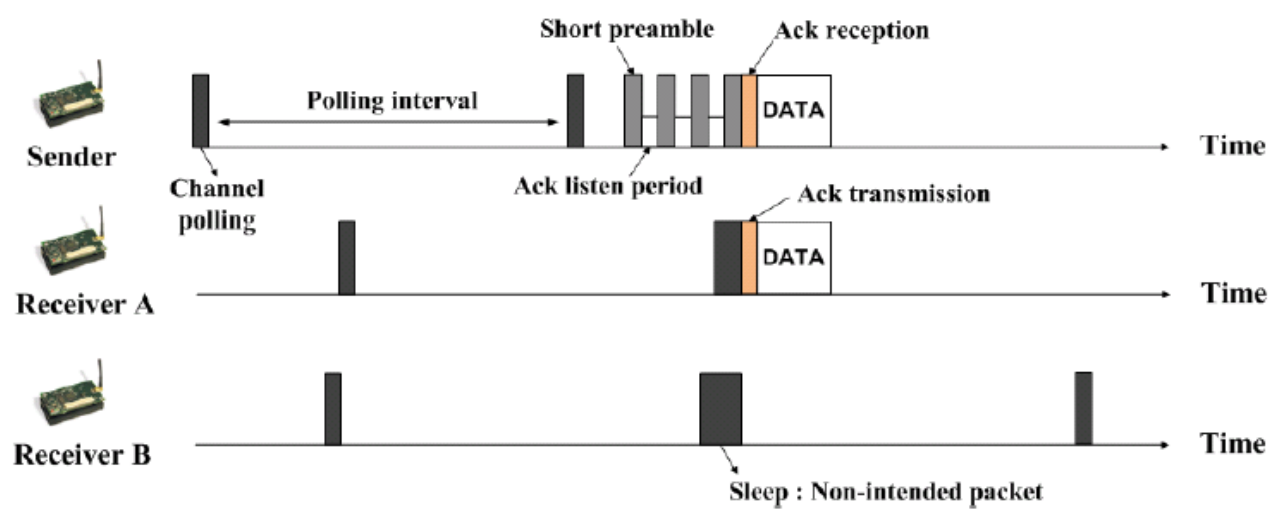

Figure 2. The basic operation of ACK-based LPL method

\subsection{The cross-technology interference}

To alliviate the CTI, several clever schemes were proposed. In [7], the authors proposed Cooperative Busy Tone (CBT) transmission scheme that can simultaneously perform channel reservation for packet transmission and transmission protection of Zigbee nodes. CBT is an innovative technique applied to PHY/MAC layer of ZigBee to inform surrounding Wi-Fi nodes of channel reservation status. A general ZigBee node transmits packets and separates a general ZigBee node for transmitting packets and a protector node for channel reservation. At the same time, a guard node informs Wi-Fi nodes that ZigBee is currently in use through a neighboring ZigBee channel. At this time, the caregiver node is compatible with a common ZigBee node and uses an XBee device capable of transmitting with higher signal strength. The reason that CBT can simultaneously reserve channels for ZigBee packet transmission is due to the structure related to ZigBee and Wi-Fi frequency configuration. One Wi-Fi channel overlaps with four ZigBee channels, one of which is used as a ZigBee data transmission channel and the adjacent channel is used as a reservation channel for sending a reservation signal to $\mathrm{Wi}-\mathrm{Fi}$. This scheme has the advantages that the reservation overhead can be reduced to a certain level by simultaneously performing the channel reservation. In addition, it increases the visibility of the ZigBee network by using the guard node capable of transmitting with high power. However, the transmission request information that the ZigBee node periodically transmits to the guardian node may not be delivered due to Wi-Fi interference, and when the channel-grunt message collides, both ZigBee and Wi-Fi nodes do not perform inefficient operation. In [8], the authros proposed a Narrow Band Protection (NBP) scheme based on signal correlation that can minimize the overhead of these channel reservation schemes. However, NBP only focuses on the ZigBee detection mechanism and cannot support a low powr operation of ZigBee networks.

\section{INTERFERENCE-AWARE CHANNEL RESERVATION SCHEME}

\subsection{Motivation}

According to the recently proposed heterogeneous wireless network studies [9], it can be seen that the 802.15.4 standard is experiencing severe performance degradation by 802.11 standard. The 802.15.4 standard transmits data at a low power of up to $1 \mathrm{~mW}$ to support energy-efficient operation. On the other hand, the 802.11 standard transmits data at a maximum power. The difference in transmit power is severely affected by the interference of the 802.11 standard because the 802.15.4 standard can not inform the surrounding 802.11 standard about the use of the network. In addition, due to the limited hardware for low power operation, even after channel preemption, it is severely interfered by the 802.11 standard. This phenomenon is defined as the Cross-Technical Interference problem.

The WSNs configured based on the PHY/MAC protocol of the IEEE 802.15.4 standard. Thus, the same type of interference suffered by the 802.15.4 standard suffers from the 802.11 standard. Recently, channel reservation schemes have been proposed for the 802.15.4 standard to minimize interference by the 802.11 standard and compete fairly for channel use. It is a technique that reserves channels for the 802.15.4 standard using a guardian, a third device that complies with the 802.15.4 standard and is capable of transmitting at power equal to the 802.11 standard. These techniques can be applied to WSNs to reduce the throughput degradation of wireless sensor networks in the presence of interference from the 802.11 standard. However, if WSNs perform low power operations, the solution cannot mitigate the performance degradation. 


\subsection{Channel reservation protocol}

In this section, we propose an intelligent channel reservation scheme to enhance the utilization of both low-power WSNs and Wi-Fi networks. We mainly focus on the asynchronous sensor MAC protocols that include basic-LPL and ACK-LPL due to their various advantages. Figure 3 illustrates the basic operation of our proposed scheme with basic-LPL. We insert the PN code in the end of the long preamble to request the reservation efficiently. This algorithm limits an excessive reservation for the entire long preamble. The protector only preempts the channel during the actual data transmission time from the Wi-Fi networks. It prevents unnecessary channel preemption and avoids significant throughput degradation of Wi-Fi networks.

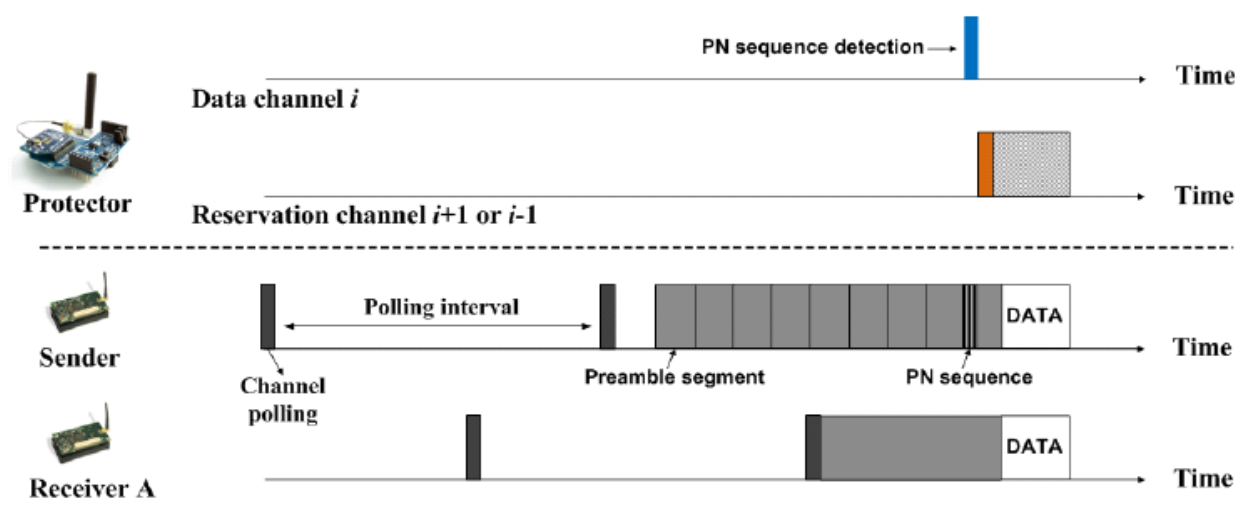

Figure 3. The basic operation of LPL scheme with an intelligent channel reservation

Our scheme uses the cross-correlation technique to share the channel reservation information. Channel reservation durations consist of $\mathrm{m}$ PN sequences. As shown in (1), the sequences have L samples. The receiver correlates a specific PN sequence with the received signal. When a signal that is matched in the correlation phase is encountered, a high spike occurs and the presence of the signal can be confirmed [11], [12].

$$
C(\Delta)=\sum_{k=1}^{L} s^{*}[k] y[k+\Delta]
$$

In case of ACK-LPL, we embed the reservation request signal in the early ACK. The field contains data packet size and the number of data. Figure 4 describes the basic operation of our proposed scheme with ACK-LPL. The protector does not need to consider short preambles of the senders. The algorithm enhances the coexistence of both networks considerably since it can intelligently avoid the CTI in the normal environment. If a PN sequence is found in the data channel, it transmits Busy Tone so that Wi-Fi can not transmit until the packet is transmitted in the reservation channel.

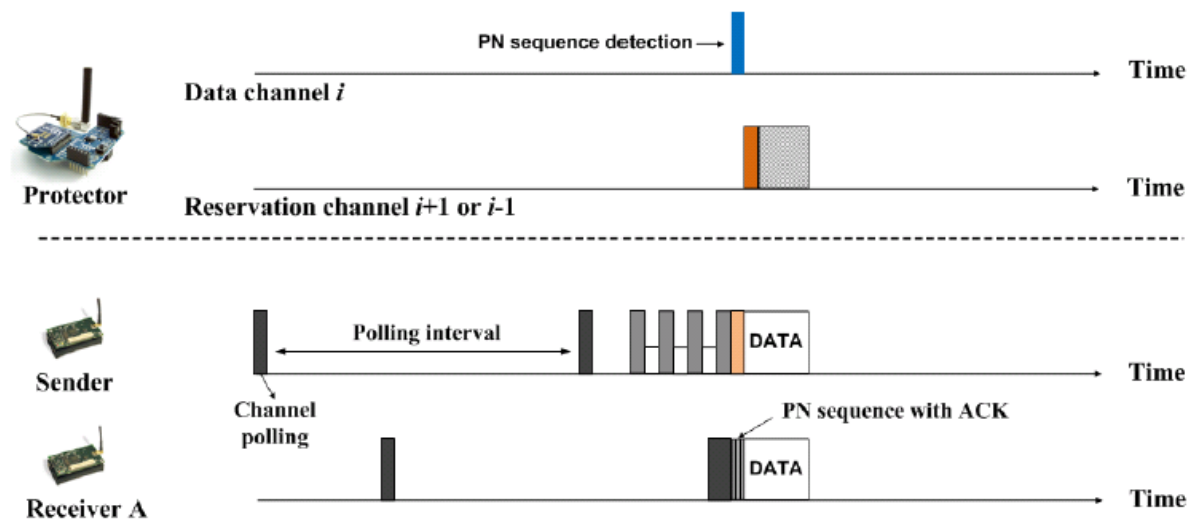

Figure 4. The basic operation of ACK-LPL scheme with an intelligent channel reservation

IACR: an interference-aware channel reservation for wireless sensor networks (Sangsoon Lim) 


\section{PERFORMANCE EVALUATION}

\subsection{Request detection rate}

In order to compare the performance of the IACR, we compared the basic ACK-LPL scheme with the discovery rate of request packets requesting data transmission. Figure 5 shows the request detection rate of the basic ACK-LPL and ACK-LPL with the IACR. We measured RDRs of both schemes. As Received Signal Strength Indicator(RSSI) decreases, decoding rate in the basic ACK-LPL is also decreased significantly. However, under various RSSIs, the detection error of the basic ACK-LPL with the IACR is much smaller than the decoding error of the basic ACK-LPL. The IEEE 802.15.4 standard specifies receiver sensitivity to $-85 \mathrm{dBm}$. While considering the receiver sensitivity, our scheme achieves higher detection accuracy at very low RSSI. Thus, our proposed scheme enhances the protection coverage at least $11 \mathrm{~dB}$ compared with the basic ACK-LPL.

In wireless sensor networks, the duty cycle interval is directly related to power consumption. In general, when the duty cycle interval is small, the RF module is turned on for a long time, which consumes more power. On the other hand, when the duty cycle interval is large, the power consumed is reduced because the RF module is turned on less time. However, when the duty cycle interval is large, the packet transmission interval increases and the overall delay tends to increase. We measured the packet transmission rate according to the duty cycle through event - based experiment. The environment assumes an environment where there are five fixed Wi-Fi clients. When one Wi-Fi AP and five Wi-Fi clients send and receive packets consecutively, it can be seen that about $25 \%$ of packets are lost in the conventional ACK-LPL method. Because the Wi-Fi clients transmit data, a reception error occurs for the preamble for data transmission of the sensor nodes. However, when we use the proposed method, we can see that the packet loss rate can be reduced to less than $10 \%$. Because we used signal correlation to tell the actual data transmission time, we could increase the packet detection rate. Packet Delivery Ratio as a function of duty cycle interval shown in Figure 6.

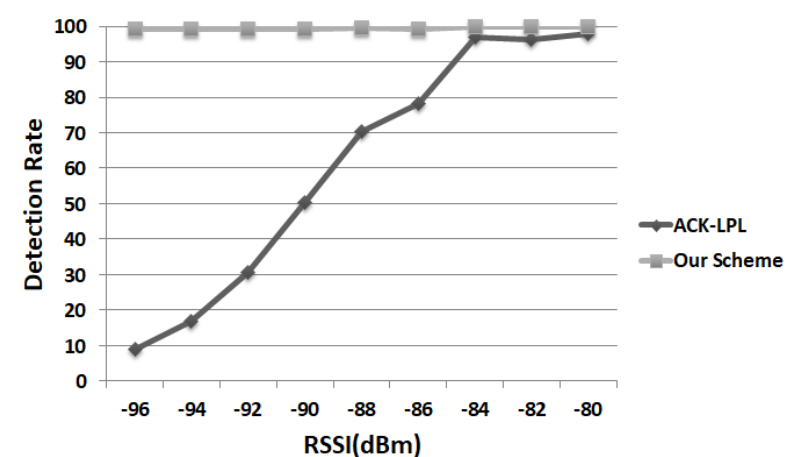

Figure 5. Request detection rate as a function of RSSI

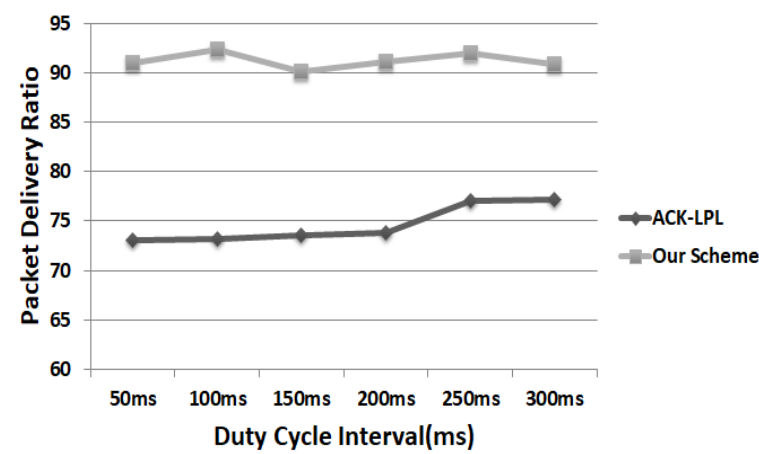

Figure 6. Packet delivery ratio as a function of duty cycle interval

\section{CONCLUSION}

In WSNs, various asynchronous low power protocols have been studied to improve energy efficiency. However, in the past, various wireless technologies have not been able to coexist, resulting in various performance degradation problems. Particularly, when the receiving node can not hear the packet of the transmitting node during the low power operation, the packet transmission rate drops sharply. To solve this problem, we propose a channel reservatino scheme considering surrounding interference. By adding signal correlation technique to the operation of existing low power wireless sensor networks, the probability of receiving control packets between sensor nodes is increased. To verify its feasibility and event-based simulation, it is proved that the packet transmission rate is higher than the existing scheme. In addition, it proves that RSSI improves overall performance by discovering control packets better than existing methods in various environments.

\section{ACKNOWLEDGEMENTS}

This work was supported by the National Research Foundation of Korea(NRF) grant funded by the Korea government(MSIT) (No. NRF-2018R1C1B5038818) 


\section{REFERENCES}

[1] Akyildiz, W. Su, Y. Sankarasubramaniam and E. Cayirci, “A Survey on Sensor Networks," IEEE Communications Magazine, Vol: 40. Issue: 8, pp. 102-114, 2002.

[2] W. Ye, J. Heidemann, and D. Estrin, “An Energy Efficient Mac Protocol for Wireless Sensor Networks,” In 21st International Annual Joint Conference of the IEEE Computer and Communications Societies, 2002.

[3] T. van Dam and K. Langendoen, "An Adaptive Energy-Effcient MAC Protocol for Wireless Sensor Networks," In Proc. of the First ACM Conference on Embedded Networked Sensor Systems, Nov.2003.

[4] J. Polastre, J. Hill, and D. Culler, "Versatile Low Power Media Access for Wireless Sensor Networks," In Proc. of The 2nd ACM SenSys Conference, Nov. 2004.

[5] M. Buettner, Gary V. Yee, E. Anderson and R. Han, "X-MAC: A Short Preamble MAC Protocol for Duty-Cycled Wireless Sensor Networks," In Proc. of The 4th ACM SenSys Conference, Nov.2006.

[6] Gollakota S, Adib F, Katabi D, Seshan S, "Clearing the RF Smog: Making 802.11 Robust to Cross-Technology Interference,” In Proceedings of ACM Sigcomm 2011

[7] Zhang X, Kang Shin G, "Enabling Coexistence of Heterogeneous Wireless Systems: case for ZigBee and Wi-Fi", In Proceedings of The 12th ACM International Symposium on Mobile Ad Hoc Networking and Computing (MobiHoc) 2011

[8] S. Lim, S. Lee, J. yoo, C. Kim, "NBP: light-weight Narrow Band Protection for ZigBee and Wi-Fi Coexistence", EURASIP JWCN 2013

[9] Huang J, Xing G, Zhou G, Zhou R, "Beyond Co-existence: Exploiting Wi-Fi White Space for Zigbee Performance Assurance," In Proceedings of The 18th IEEE International Conference on Network Protocols (ICNP) 2010

[10] Sangsoon Lim, Hayoung Oh, "EETA: An Energy Efficient Transmission Alignment for Wireless Sensor Network Applications", International Journal of Electrical and Computer Engineering (IJECE), Vol. 6, No 5. October 2016

[11] Gollakota S, Katabi D, “Zig-Zag Decoding: combating hidden terminals in wireless networks", In Proceedings of ACM Sigcomm 2008

[12] Sen S, Choudury RR, Nelakuditi S, "CSMA/CN: Carrier Sense Multiple Access with Collision Notification," In Proceedings of ACM MobiCom 2010 